\title{
A Flexible Accession for Brain Tumour Detection and Classification using Al Methodologies: Survey
}

\author{
$V$ Ramya Manaswi ${ }^{1,{ }^{*}}$ and $B$ Sankarababu ${ }^{2}$ \\ ${ }^{1}$ MTech Student, Computer Science and Engineering, GRIET, Hyderabad, Telangana, India. \\ ${ }^{2}$ Professor, Computer Science and Engineering, GRIET, Hyderabad, Telangana, India.
}

\begin{abstract}
In order to get a successful and appropriate treatment for the disorder regarding health, précised and identifying it early is much important in the scenario of brain tumor treatment. Prior knowledge and detection of the tumor helps to cope up with good medication, and also helps in saving a life in due time. Bio-medical informatics(BI) and Computer aided diagnosis(CAD) are benefiting neurooncologists in many ways. Machine learning algorithms are now used to do Image processing on medical images and contrast with the information due to manual diagnosis of Brain tumor which is always a tedious task because of human error is indulged. When compared with manual traditional practices, Computer aided mechanisms are compared to obtain better results. In this paper we are presenting the existing models or architectures overview of various researchers who dedicatedly addressed and worked on this tedious task.
\end{abstract}

Keywords - Random forest (RF), Expectation-maximization(EM), Decision Tree(DT), CNN, DNN

\section{Introduction}

Medical Image processing and segmentation for tumour detection in brain using the Magnetic-resonanceImages(MRI) in some cases uses different medical imaging modes which are very keen in process of deciding rights in MRI most notably, Support Vector Machine (SVM), Fuzzy Clustering Means (FCM), Artificial Neural Network (ANN), Neural Networks(NN) and expectationmaximization(EM) based algorithmic approaches which are some of the popular techniques used for region-based segmentation and so to extract the important information from the medical image processing. Lately, the presentation of advancements in IT and e-medical supervision system in healthcare area assists medical specialists with giving better medical treatment to the suffering patients. This examination tends to the issues with segmentation of abdominal cerebrum tissues and ordinary tissues like cerebrospinal liquid (CSL), white matter (WM), and grey matter (GM) from MRI by implementing feature extraction and SVM classifier.

. BT generally originate because of the rapid growth of the cells and when the situation is not controllable too. The impact of BT will be different in different age groups. BT expansion is generally dependent on the lifestyle, gender etc. Because BT cases were growing day by day and the impact of BT is more on the people, there were many people who are losing their lives which is uncontrollable. Shockingly there are many children who are been affected by the BT and loosing lives. The death rate is growing in children these days. So, in order to control these situations under control there must be an innovative way to predict the BT and to cure the BT in the early stages. So, these conditions made to focus on the proposed system.

Hydrogen molecules are regularly utilized in clinical and research MRIs to produce a distinguishable radiofrequency signal that receiving wires get right upfront closeness to the life structures being inspected. Hydrogen molecules normally exist in wealth in people and other natural organic entities, particularly in fat and water. By using the data mining strategies, huge relations and examples from the information can be separated. The procedures of ML (AI) and Data mining are as a rule adequately utilized for BTD and prevention at a beginning phase.

In this work, we present a programmed Brain Tumour Segmentation (BTS) procedure dependent on CNN. We have utilized three MRI perspectives on human cerebrum. $\mathrm{X}$-ray check is utilized on the grounds that it is not so much destructive but rather more exact than CT cerebrum check. All past deals with the dataset that we are working with are for arrangement of tumour types. None of the past works

\footnotetext{
*Corresponding author: m.srividya1998@gmail.com
} 
performed on this dataset are planned for Segmentation. The principal commitment of our paper is the dividing of the pictures in light of the course of caught MR pictures. Subsequently, three networks will be trained independently to accomplish better segmentation results

\section{Literature Review}

Zahra Sobhaninia et al., proposed brain tumour identification requires high precision, where minute mistakes in judgment may prompt disaster. Thus, Brain tumour Segmentation is a significant test for clinical purposes. At present a few techniques exist for tumour segmentation however they all need high precision. Here we present an answer for Brain tumour segmenting by utilizing deep learning techniques. In this work, we considered various points of cerebrum MR pictures and ap plied various organizations for division. The impact of utilizing separate networks for segmentation of MR pictures is assessed by contrasting the outcomes and a single network. Exploratory assessments of the organizations show that Dice score of 0.73 is accomplished for a single network and 0.79 in got for various networks (1)

Masoumeh Siar et al., proposed Cerebrum tumour can be grouped into two kinds: generous and harmful. Ideal and prior recognition and treatment plan prompts improved treatment and expanded the lifespan of patients. Quite possibly the most prominent and significant techniques is to utilize Deep Neural Network (DNN). To identify a tumour cerebrum Magnetic Resonance Imaging (MRI)pictures fed to the CNN model. Pictures were first embedded into the Convolutional Neural Network. The achieved accuracy from the SoftMax function and Fully Connected layers using MRI scanned images acquired $98.06 \%$. Likewise, the precision of Convolutional Neural Network is acquired with the Radial-Basis-Function(RBF) model is $97.03 \%$ also used Decision Tree (DT)and achieved $94.42 \%$. Not-with-standing on the exactness basis, we utilize the Sensitivity benchmarking, precision, and specificity assess the execution of network. As per the outcomes got from the above classifiers, the SoftMax function got the best precision using CNN (2)

Tonmoy Hossain1 et al., proposed brain tumour segmentation is perhaps the most important and burdensome aspects in the territory of image processing on medical images as a human-helped manual grouping can bring about erroneous expectation and determination. In addition, it is a disturbing assignment when there is a lot of information present to be helped. Mind tumours have high variety apparently and there is a comparability among tumour and ordinary tissues and subsequently the extraction of tumour locales from pictures gets unflinching. The authors proposed a strategy to isolate tumour from 2D MRI brain Images using "Fuzzy C-Means clustering algorithm" also trained using conventional classifier algorithms and CNN. The experimentation was carried on a continuous data with assorted tumour size, area, shape, and distinctive picture forces. In conventional classifier algorithms, applied 6 customary algorithms to be specific K-Nearest Neighbour(KNN), Logistic Regression(LR), Multilayer Perceptron(MLP), Support Vector Machine(SVM), Naïve Bayes(NB) and Random Forest(RF) which was carried out in scikitlearn. A short time later, we proceeded onward to $\mathrm{CNN}$ is executed utilizing Tensor flow and Keras on the grounds that it respects a preferred presentation over the conventional. Author mentioned in his paper CNN acquired a $97.70 \%$ precision, which is exceptionally convincing. The primary point of this paper is to recognize typical and unusual pixels, in view of surface based and factual based highlights. Also achieved $92.24 \%$ and $97.80 \%$ precision utilizing SVM and CNN. (3)

G. Hemanth et al., proposed the brain tumour(BT) recognition which has turned up as an most difficult in the field of medicine. BT can be indicated as a distorted growth of tissue in which the cells duplicate unexpectedly and interminably. The cycle of Image Segmentation is received for extricating strange tumour locale inside the cerebrum. In the MRI (Magnetic Resonance Image) Segmentation of mind tissue holds extremely huge to determine the presence of layouts concerning the cerebrum tumour. There is a huge concealed data is captured and saved in the Health care domains. With legitimate use of exact information mining order methodologies, early conjecture of any contamination can be performed effectively. Predominant piece of which is embraced feasibly. The assessment investigations summary of danger factors which are being takes place while doing Brain tumor observation systems. additionally, the proposed technique certifications to be incredibly compelling and precise for Brain tumour detection, classification and segmentation. In order to get highly accurate automation and semi automation systems are very useful. The researcher proposed a programmed segmentation methodology which relies on CNN using small kernels which size is $3 \times 3$. By consolidating this single strategy, segmentation and classification is refined. One of the Neural Network based technique called CNN which has layer-based classification of results. The methods of AI with Data mining are adequately utilized for Brain tumour detection and avoidance at a beginning phase. This accomplishes $92.7 \%$ precision utilizing CNN (4)

S. Somasundaram et al., proposed Basic segment in diagnosing tumour, planning treatment and building up a result for assessing Brain tumour segmentation should have been profoundly exact and solid. MRI scans help and 
guiding the medical domain experts to recognize the extremely minor unusual development in human beings body. The Deep Neural Networks (DNNs) and AI methods have great accomplishment in 2D picture segmentation, however it's a moving difficult for DNNs to section basic organ parts using 3D clinical MRI scan reports. Segmentation relating tumour identification incorporates a few preparing strategies that are sorted into segmentation. research centres essentially around 3D ANN, SVM, CNN and Multi class SVM to do in précised Segmentation process. To eliminate computational weight of preparing 3D clinical sweeps, this research intends to survey the present advancement in segmentation of image and classification dependent on productive and compelling towards handling of tumour contaminated human mind MRI adjoining picture fixes that can go through the network with an objective of tumour, while mechanically adjusting towards an awkwardness there in the information At last, this article inferring about advancements on Detection and segmentation of tumour-based picture preparing for DL models. Analysis results of the underlying dataset show that the streamlining through GA approach has improved the complete exactness of SVM from $79.4 \%$ to $91.6 \%$ and of ANN from $75.5 \%$ to $94.8 \%$ (5)

Bhagyashri $H$. et al., proposed a model for theneed to create computer analysis frameworks for analysis of Brain tumour. Brain tumour(BT) identification at beginning phase has gotten vital. In experimentation, cerebrum tumour MRI are utilized to recognize and characterize the dangerous and kind-hearted mind tumours. X-ray pictures which are collected by Brats dataset collected in 2015 by MICCAI. BTs are divided by utilizing image-processing methodologies. To de feature extraction of images the shape-based highlights are utilized. Removed shape-based highlights are taken care of AI algorithms as SVM and RF algorithms to order amiable and harmful cerebrum tumours. It accomplished the precision for irregular timberland is $86.66 \%$ (6)

Madhu Priya G et al., proposed a model with help of the MRI pictures. The process of segmentation was performed and the segmented images will be capable to measure up to the normal BT tissues and cells. The results are shown in the perspective of correlation. The researcher in his work done on segmentation to utilize a probabilistic neural network(PNN) and convolutional neural network(CNN).
The design of $\mathrm{CNN}$ which has both $3 * 3$ and $7 * 7$ layers to build a cascaded architecture, in order to do a segmentation of tumour accurately in a powerful way, on Brats13 MRI dataset. Same way they utilized a PNN model to identifying tumours and analyse the problems on both models. SO, they came up with an exceptional PNN and CNN models to utilize image processing and computer vision(CV) strategies. the model arrangements with both local and global highlights. At last, got the yield fragmented picture just as examined precision and loss of our models. At long last, got the yield segmented picture just as investigated exactness and loss of our models (7)

K. Abbas et al., proposed a model to predict the BT using unpredictable design and its converging with the ordinary cells, and the arrangement of tumour from typical tissues was a difficult errand. The author in his research have brought up with a philosophy called LIPC in order to do the classification and segmentation of a brain tumour. The primary step in his research is to solve the multiclass image segmentation as a grouping task. X-ray groupings of the cerebrum have normal qualities (area) and are scanty (sparsity). Author used both these parameters in his proposed system. Firstly they focussed on image preprocessing strategies are utilized for image enhancement and noise removal. After 12 textural diverse features were determined and diminished because of PCA to do a better classification. 2013 MICCAI dataset is used in this experiment which had a low-level-tumour to high-leveltumour patient's data. This technique introduced a dice Score which is 0.95 for complete tumour which is a long shot contrasted with the strategies effectively accessible as far as dice Score and time complexity (8)

Zheshu Jia et al., discussed about the detection of brain unusual structures by essential imaging procedures which are very challenging. So, the author proposed a model called Fully-Automatic Heterogeneous Segmentation(FAHS) along with Support Vector Machine(SVM) was proposed to detect and separate tumour in MRI scans of brain images, depends on DL strategies(11). The proposed system shows the partition of the entire cerebral venous framework into MRI imaging with the expansion of another FAHS algorithm depends on underlying, morphological subtleties. The fragmenting capacity is recognized using uniformity which is high in level between present and neighboring tissues. ELM is an another algorithm for learning to comprise of at least one layer of hidden layers. Such algorithms are utilized in 
different areas, which includes classification and regression. In MRI pictures, the PNN grouping framework which was used for training and testing the exactness of tumour identification on dataset images. The mathematical outcomes show practically $98.51 \%$ precision in recognizing strange and typical tissue from cerebrum Magnetic Resonance image that exhibit the productivity of the framework recommended (9)

Deepali Vikram Gore1 et al., proposed One in perilous infections to identify is cerebrum tumour. Utilizing deep learning methods, has shown decrease of mistake in human mid 99 determination of the sickness. Particularly, diagnosis of brain tumour requires high precision, where minute mistakes in analysis may prompt confusions. In medical image processing, cerebrum tumour exposure stays a requesting position. It is a complicated task to detect a tumour in brain. A few commotions also postpone influences the picture exactness. Picture division and MRI methods have become an accommodating clinical diagnostic instrument for the assessment of the mind and other clinical dataset. Segmentation of an image is a powerful space of Medical Image Processing(MIP). This process is carried out to draw out the various jobs from clinical pictures like CT, MRI check, also includes Mammography, and so on. The researcher, we introduced a deliberate training of brain tumour utilizing deep learning methods. The examination and relative investigation of late information connected with cerebrum problem discovery utilizing Deep Neural Networks is considered in this audit. The result of this paper expresses the different exploration holes distinguished from the writing audit. The exactness accomplished is $88.9 \%$. (10)

\section{Comparison}

Following table is the comparison between the models I have surveyed.

Table 1. Comparison of the Methods used

\begin{tabular}{|c|c|c|c|c|}
\hline Year & Author & $\begin{array}{l}\text { Algorithm/ } \\
\text { System }\end{array}$ & $\begin{array}{l}\text { Accurac } \\
\mathrm{y}\end{array}$ & Ref. \\
\hline 2018 & $\begin{array}{l}\text { Zahra } \\
\text { Sobhanin } \\
\text { ia et al., }\end{array}$ & $\begin{array}{l}\text { Deep learning } \\
\text { techniques }\end{array}$ & $\begin{array}{l}\text { Dice } \\
\text { score } \\
\text { for one } \\
\text { network } \\
-73 \% \\
\text { For } \\
\text { multiple } \\
\text { network } \\
\text { s- } \\
79 \%\end{array}$ & [1] \\
\hline
\end{tabular}

\begin{tabular}{|c|c|c|c|c|}
\hline 2019 & $\begin{array}{l}\text { Masoum } \\
\text { eh Siar et } \\
\text { al }\end{array}$ & $\begin{array}{l}\text { FCNN, } \\
\text { RBF, } \\
\text { DT }\end{array}$ & $\begin{array}{c}98.06 \%, \\
97.03 \%, \\
94.42 \%\end{array}$ & [2] \\
\hline 2019 & $\begin{array}{l}\text { Tonmoy } \\
\text { Hossain1 } \\
\text { et al., }\end{array}$ & $\mathrm{CNN}$ & $97.70 \%$ & [3] \\
\hline 2019 & $\begin{array}{l}\text { G. } \\
\text { Hemanth } \\
\text { et al., }\end{array}$ & $\mathrm{CNN}$ & $92.56 \%$ & [4] \\
\hline 2019 & $\begin{array}{l}\text { S. } \\
\text { Somasun } \\
\text { daram et } \\
\text { al., }\end{array}$ & $\begin{array}{l}\text { SVM, } \\
\text { ANN }\end{array}$ & $\begin{array}{l}91.6 \% \\
94.82 \%\end{array}$ & [5] \\
\hline 2019 & $\begin{array}{l}\text { Bhagyas } \\
\text { hri H. et } \\
\text { al., }\end{array}$ & $\begin{array}{l}\text { SVM, } \\
\text { RF }\end{array}$ & $86.66 \%$ & [6] \\
\hline 2019 & $\begin{array}{l}\text { Madhu } \\
\text { Priya G } \\
\text { et al., }\end{array}$ & $\begin{array}{l}\text { PNN, } \\
\text { CNN }\end{array}$ & - & [7] \\
\hline 2019 & $\begin{array}{l}\text { K. Abbas } \\
\text { et al. }\end{array}$ & $\begin{array}{l}\text { Deep learning } \\
\text { techniques }\end{array}$ & $\begin{array}{l}\text { Dice } \\
\text { score- } \\
95 \%\end{array}$ & [8] \\
\hline 2020 & $\begin{array}{l}\text { Zheshu } \\
\text { Jia et al., }\end{array}$ & FAHS-SVM & $98.51 \%$ & [9] \\
\hline 2020 & $\begin{array}{l}\text { Deepali } \\
\text { Vikram } \\
\text { Gore1 et }\end{array}$ & DNN & $88.66 \%$ & [10] \\
\hline
\end{tabular}

\section{Conclusion}

In this paper a partial survey for brain tumour detection is done. Various techniques which are proposed earlier are mentioned here along with their usage of algorithms and assumption for execution of the problem. The dataset used for different technique is also mentioned and the results obtained by using those techniques are mentioned. This work can be used for extension of those techniques and for obtaining better results by future enhancements.

\section{References}

1 Z. Sobhaninia, S. Rezaei, A. Noroozi, M. Ahmadi, H. Zarrabi, N. Karimi, A. Emami, and S. Samavi, arXiv [cs.CV] (2018).

2 M. Siar and M. Teshnehlab, 2019 9th International Conference on Computer and Knowledge 
Engineering (ICCKE) (2019)

3 T. Hossain, F. S. Shishir, M. Ashraf, M. D. A. Al Nasim, and F. Muhammad Shah, in 2019 1st International Conference on Advances in Science, Engineering and Robotics Technology (ICASERT) (2019), pp. 1-6.

4 G. Hemanth, M. Janardhan, and L. Sujihelen, in 2019 3rd International Conference on Trends in Electronics and Informatics (ICOEI) (2019), pp. 1289-1294

5 S. Somasundaram and R. Gobinath, 2019 International Conference on Machine Learning, Big Data, Cloud and Parallel Computing (COMITCon) (2019)

6 B. H. Asodekar, S. A. Gore, and A. D. Thakare, in 2019 5th International Conference on Computing,
Communication, Control And Automation (ICCUBEA) (2019), pp. 1-5

7 G. Madhupriya, N. M. Guru, S. Praveen, and B. Nivetha, in 2019 3rd International Conference on Trends in Electronics and Informatics (ICOEI) (2019), pp. 758-763

8 K. Abbas, P. W. Khan, K. T. Ahmed, and W.-C. Song, in 2019 International Conference on Information and Communication Technology Convergence (ICTC) (2019), pp. 531-536.

9 Z. Jia and D. Chen, IEEE Access 1 (2020)

10 D. V. Gore and V. Deshpande, in 2020 International Conference for Emerging Technology (INCET) (2020), pp. 1-4.

11 C. U. Kumari, S. Jeevan Prasad, and G. Mounika, 2019 3rd International Conference on Computing Methodologies and Communication (ICCMC) (2019). 